\section{Impeding deposition}

Lewy bodies and other types of a-synuclein-rich pathology are typically associated with Parkinson disease but can also be found in many cases of Alzheimer disease (AD). Several studies have indicated that $\alpha$-synuclein and amyloid- $\beta(\mathrm{A} \beta)-$ the principal component of one of the hallmark pathologies of $\mathrm{AD}$, namely amyloid plaques - interact, but it is unknown whether $\alpha$-synuclein affects $\mathrm{A} \beta$ pathology in vivo. Now, Bachhuber et al. show in mice that $\alpha$-synuclein inhibits amyloid plaque formation and, as a result, might exacerbate synaptic pathology.

Previous studies have found that fibrils of $\alpha$-synuclein could induce the aggregation of tau, another protein implicated in several neurodegenerative diseases. Thus, the authors examined whether $\alpha$-synuclein could similarly 'cross-seed' amyloid plaque formation. To do so, they used mice that expressed mutant forms of amyloid precursor protein (APP; from which $A \beta$ is derived) and presenilin 1 (which is involved in APP processing) in neurons and eventually developed amyloid plaque pathology (APPPS1 mice), and mice that expressed a mutant form of $\alpha$-synuclein and eventually developed Lewy body pathology ((A30P)aSYN mice).

The authors intracerebrally injected 6-week-old, pre-pathology APPPS1 mice with brain homogenate from wild-type mice, APPPS1 mice with amyloid plaques or (A30P)aSYN mice with $\alpha$-synuclein pathology. They found that only the APPPS1 homogenate induced plaque formation in the hippocampus, indicating that $\alpha$-synuclein does not initiate plaque formation.

To examine whether $\alpha$-synuclein may have some other role in plaque development, the authors crossed the APPPS1 and (A30P)aSYN mice to generate 'double-transgenic' animals. These double-transgenic mice exhibited reduced hippocampal plaque load compared with APPPS1 mice at 4 months of age, although both groups had similar brain levels of APP and the two main forms of A $\beta$.

The findings described above suggest that $\alpha$-synuclein might in fact impair plaque formation. In further support of this assertion, cerebral injection of brain homogenate from plaque-harbouring APPPS1 mice induced markedly less hippocampal plaque formation in the double-transgenic mice than in the APPPS1 mice. Moreover, in APPPS1 mice, injection of a mixture of brain homogenates from plaque-containing APPPS1 and (A30P)aSYN mice induced considerably less cross-seeding than did injection of APPPS1 brain homogenate. Finally, frontal cortex homogenates from patients with $\mathrm{AD}$ who had plaque pathology induced much more seeding in APPPS1 mice than did those from patients with dementia with Lewy bodies (DLB), which is usually associated with both $A \beta$ and a-synuclein pathology.

The authors had previously shown that $A \beta$ was able to infiltrate and form deposits in grafted wild-type cells in mice. Here, they introduced

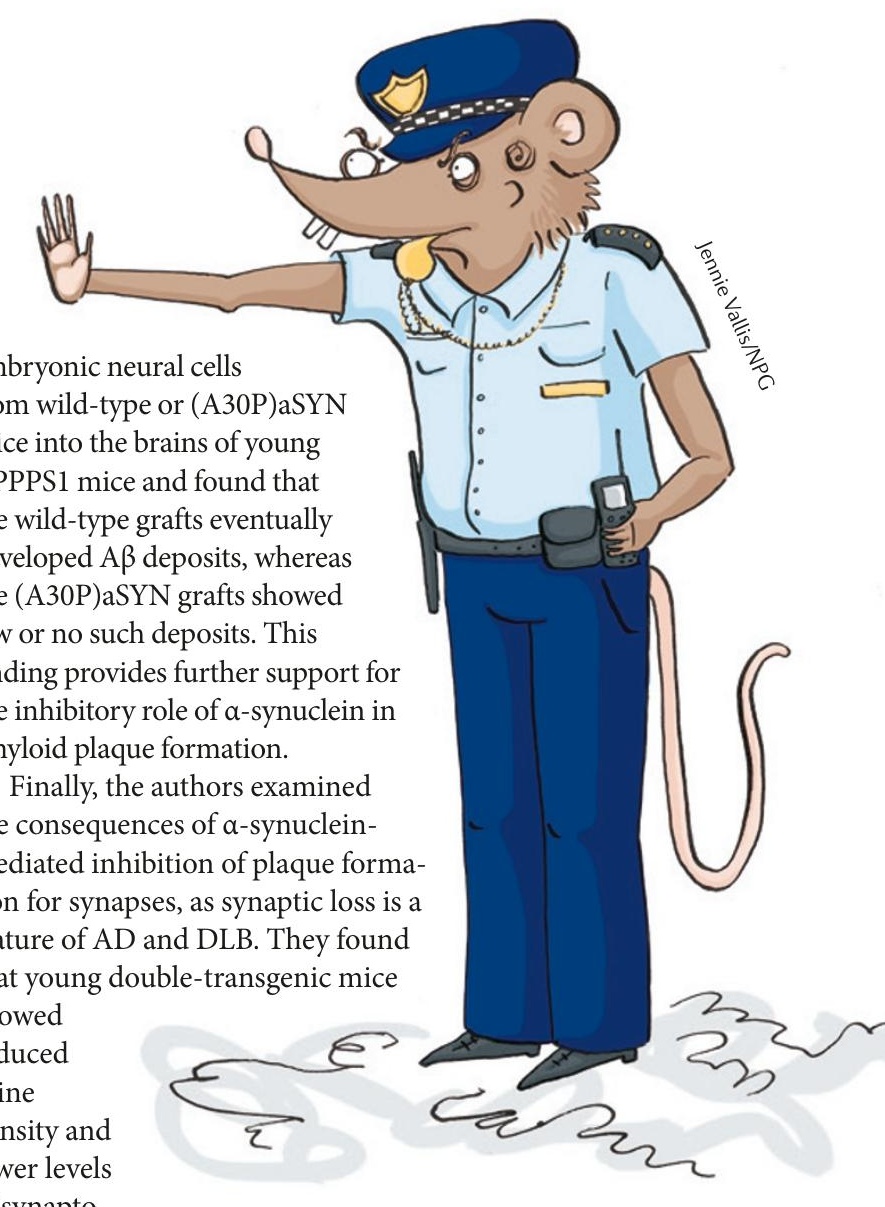
of synapto-

physin (a marker of synapses) compared with young APPPS1 or (A30P)aSYN mice, suggesting that the presence of both $\alpha$-synuclein and $\mathrm{A} \beta$ might exacerbate synaptic pathology.

Together, these findings show that $\alpha$-synuclein can inhibit $A \beta$ deposition and hence plaque formation. Whether this effect results from a direct interaction between these proteins remains unclear, and the consequences of their interaction require further investigation.

Darran Yates

\section{ORIGINAL RESEARCH PAPER}

Bachhuber, T. et al. Inhibition of amyloid- $\beta$ plaque formation by $\alpha$-synuclein. Nat. Med. 21, 802-807 (2015)

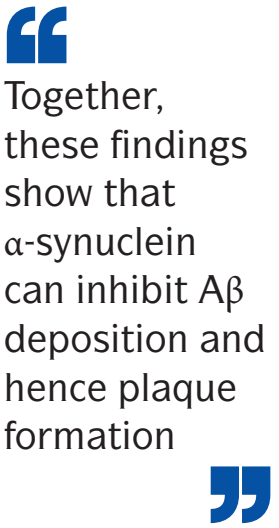

